\title{
Setting traps for NKG2A gives NK cell immunotherapy a fighting chance
}

\author{
Frank Cichocki and Jeffrey S. Miller \\ Department of Medicine, Masonic Cancer Center, University of Minnesota, Minneapolis, Minnesota, USA.
}

\begin{abstract}
The equilibrium of signaling through activating and inhibitory receptors dictates whether a given NK cell will execute cellular cytotoxicity. In this issue of the $J \mathrm{Cl}$, Kamiya et al. describe a novel approach to efficiently inhibiting surface expression of the inhibitory receptor CD94/NK group 2 member $A$ (NKC2A) through retention of the protein in the endoplasmic reticulum. In adoptive transfer experiments into tumor-bearing immunodeficient mice, NKG2 $A^{\text {null }}$ NK cells were significantly more effective at eliminating HLA-E-expressing tumor cells than NKG2A+ NK cells. This study provides proof of concept for a new immunotherapeutic approach using NKG2A ${ }^{\text {null }}$ NK cells.
\end{abstract}

\section{A central role for NKG2A} in NK cell inhibition

CD94/NK group 2 member A (NKG2A) and NKG2C were identified in the mid1990s as cell surface glycoproteins that form disulfide-bonded heterodimers with CD94 and bind the nonclassical MHC class Ib molecule HLA-E. NKG2C engagement in CMV-seropositive individuals imparts NK cell activation (with activated cells termed adaptive NK cells), while engagement of CD94/NKG2A transduces an inhibitory signal, consistent with the presence of two I/VxYxxL immunoreceptor tyrosine-based inhibitory motifs (ITIMs) within the cytoplasmic domain of NKG2A (1-4). While NKG2A expression and NKG2C expression are usually mutually exclusive, the frequency of $\mathrm{NKG}_{2} \mathrm{~A}^{+} \mathrm{NK}$ cells is considerably higher than that of $\mathrm{NKG}_{2} \mathrm{C}^{+} \mathrm{NK}$ cells, especially in CMV-seronegative donors (5). Therefore, alongside killer immunoglobulin-like receptors (KIR), NKG2A represents a dominant inhibitory receptor on NK cells. The NKG2A phospho-ITIMs inter- act directly with the $\mathrm{SH} 2$ domains of the tyrosine phosphatases SHP-1 and SHP-2 (6). One of the major targets of SHP-1mediated dephosphorylation in NK cells is the guanine exchange factor and adaptor protein Vav1. Dephosphorylation of Vav1 prevents Rac1-dependent rearrangement of the actin cytoskeleton and amplification of activating signals (7). Engagement of CD94/NKG2A by HLA-E within inhibitory signaling clusters can also lead to the phosphorylation of the signaling adaptor protein Crk and disruption of actindependent signaling upstream of Vav1 (8). NKG2A is uniformly high on immunoregulatory $\mathrm{CD} 56^{\text {bright }} \mathrm{NK}$ cells, whereas cytotoxic CD56 $^{\text {dim }}$ NK cells exhibit more heterogeneous expression, with a general decrease associated with terminal differentiation (9).

HLA-E molecules are expressed at low levels in most tissues and primarily present peptides derived from the leader sequences of classical class Ia HLA molecules (10). However, HLA-E is commonly expressed at high levels on the surface of a variety

\section{Related Article: p. 2094}

Conflict of interest: JSM serves on the scientific advisory board of, and consults for, GT BioPharma Inc. and Fate Therapeutics. He has received research funds from these relationships. He also serves on the scientific advisory boards for CytoSen Therapeutics and Onkimmune. FC consults for Fate Therapeutics and has received research funds from this relationship.

Copyright: () 2019, American Society for Clinical Investigation.

Reference information: / Clin Invest. 2019;129(5):1839-1841. https://doi.org/10.1172/JCI128480. 
A

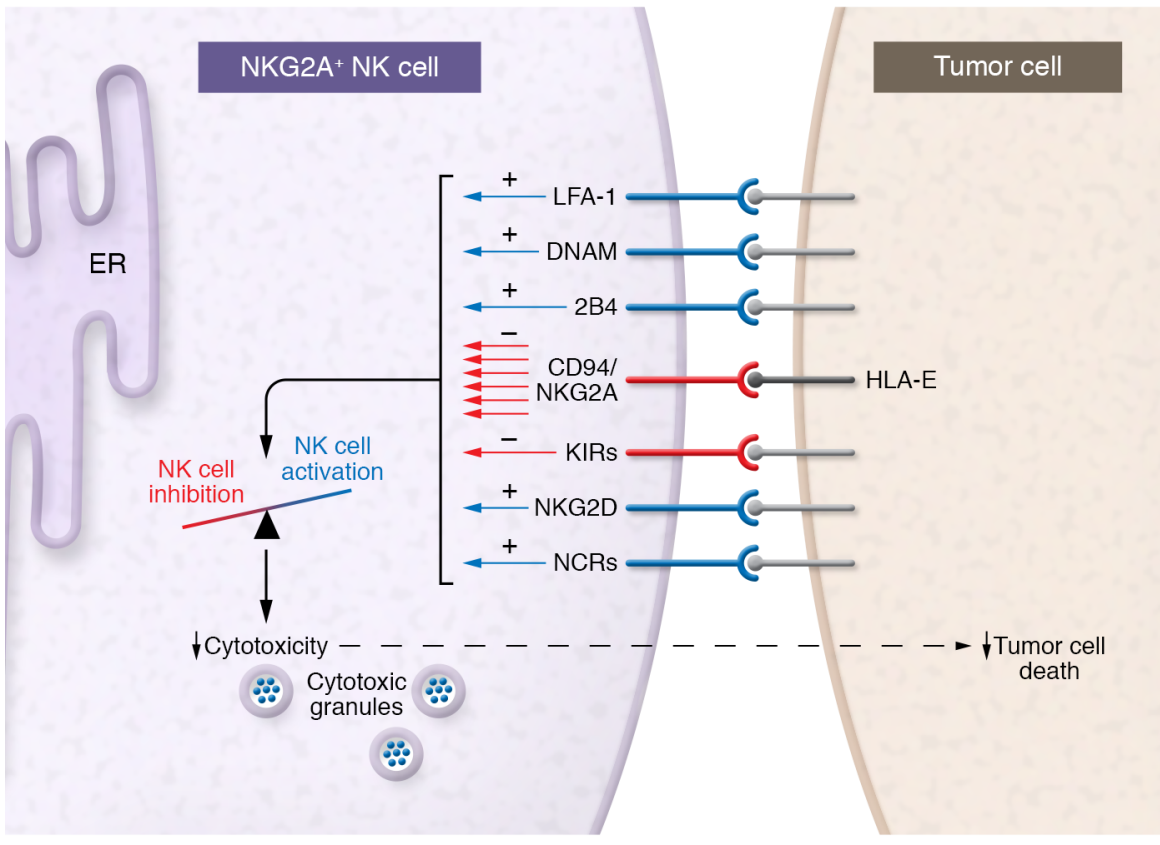

B

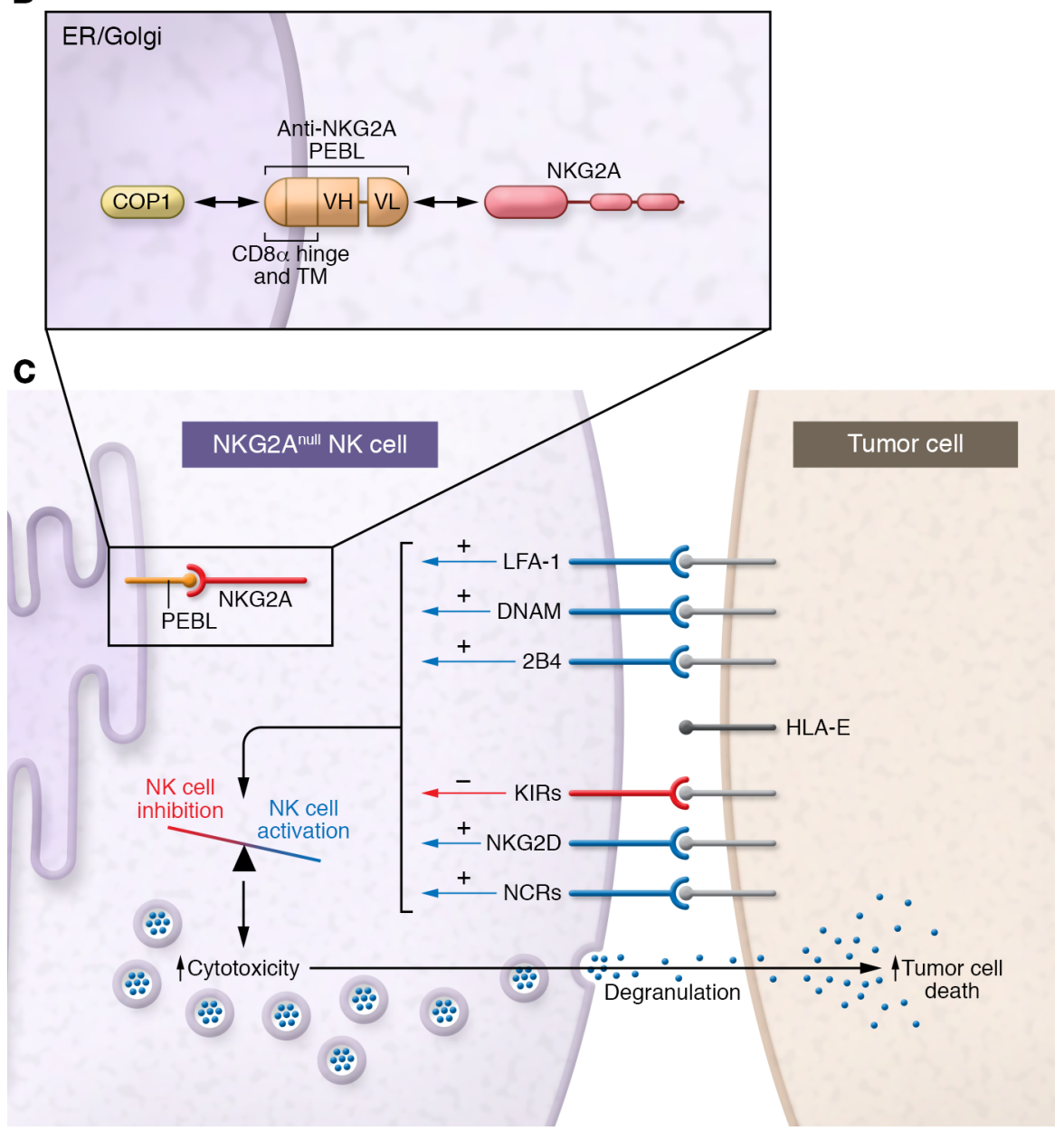

Figure 1. Trapping NIKC2A in the ER enhances NK cell cytotoxicity against HLA-E-expressing tumors. (A) High HLA-E+ tumors deliver a potent inhibitory signal that leads to inhibition of NK cell function (five relative activating signal units vs. seven inhibitory). (B) Kamiya et al. developed a series of NKG2A PEBLs consisting of an scFv derived from an anti-NKG2A antibody linked to ER-retention domains. Transduction of human peripheral blood NK cells with retrovirus containing PEBL cassettes leads to efficient intracellular retention of NKC2A. (C) After NKC2A knockdown by PEBLs, the resulting NKC2A ${ }^{\text {null }}$ NK cells exhibit greater cytotoxicity against HLA-E-expressing tumor cells due to a net lack of inhibitory signaling (five relative activating signal units vs. one inhibitory).

hinge and transmembrane domain, allowing for direct binding to the ER protein COP1, almost completely blocked NKG2A surface expression (Figure 1B).

After confirming that no other NK cell receptors were altered in response to NKG2A PEBL transduction, the authors generated multiple tumor cell lines with strong NKG2A-binding potential by transducing them with HLA-E plus HLA-G signal peptide. Relative to control $\mathrm{NKG} \mathrm{A}^{+}$ NK cells (expressing only GFP), NKG2A null NK cells (NKG2A PEBL-transduced) exhibited markedly higher cytotoxicity against these HLA-E-overexpressing lines. Therefore, the balance in signaling inputs was tipped; $\mathrm{NKG}_{2} \mathrm{~A}^{+} \mathrm{NK}$ cells that received a negative signal in response to HLA-E ligation were restrained in their killing (Figure 1A), while activating signals prevailed in NKG2A $\mathrm{A}^{\text {null }}$ cells to trigger cytotoxicity (Figure 1C). The authors also tested the function of control and $\mathrm{NKG} \mathrm{A}^{\text {null }} \mathrm{NK}$ cells against tumor cell lines with endogenous HLA-E expression that could be further elevated after exposure to IFN- $\gamma$. Again, NKG2A ${ }^{\text {null }}$ NK cells exerted significantly higher cytotoxicity with or without the addition of IFN- $\gamma$ in three out of the four lines tested. Importantly, more robust cytotoxicity was also observed by NKG2A $\mathrm{A}^{\text {null }} \mathrm{NK}$ cells in response to acute myeloid leukemia (AML) specimens that were collected from patient bone marrow and treated with IFN- $\gamma$.

Kamiya et al. wrap up this study with xenogeneic adoptive transfer experiments designed to assess the antitumor capabilities of NKG2A $\mathrm{A}^{\text {null }}$ NK cells in vivo. They engrafted the Ewing sarcoma cell line 
ES8 or the osteosarcoma line U2OS (both transduced with HLA-E plus HLA-G signal peptide) into immunodeficient mice. Mice were then given two infusions of either control $\mathrm{NKG}_{2} \mathrm{~A}^{+} \mathrm{NK}$ cells or $\mathrm{NKG}_{2} \mathrm{~A}^{\text {null }}$ NK cells at days 1 and 5 after tumor injection along with IL-2 (3 times per week). In both tumor models, control $\mathrm{NKG}_{2} \mathrm{~A}^{+} \mathrm{NK}$ cells were effective only at delaying tumor development, while the administration of NKG2A ${ }^{\text {null }}$ NK cells resulted in long-term survival for most mice. These impressive in vivo results provide proof of concept for the use of NKG2A ${ }^{\text {null }} \mathrm{NK}$ cells as a novel immunotherapy for treating patients with cancer.

\section{Future directions}

There are three ways to block or diminish NKG2A inhibition: Trapping NKG2A as described here, CMV exposure to bias the repertoire towards $\mathrm{NKG}_{2} \mathrm{C}^{+} / \mathrm{NKG} 2 \mathrm{~A}^{\text {low/- }}$ adaptive NK cells, and with therapeutic antibody blockade. Two recent publications in Cell $(19,20)$ demonstrate the beneficial impact of targeting NKG2A with blocking antibodies for enhanced cytotoxic immune response against cancer. André et al. recently reported results from a phase 2 trial using monalizumab, a humanized anti-NKG2A antibody, in combination with the antiEGFR antibody cetuximab in previously treated squamous cell carcinoma patients. They observed a $31 \%$ objective response rate in this patient population (20). van Montfoort et al. showed that antibody blockade of NKG2A potentiated $\mathrm{CD}^{+} \mathrm{T}$ cell responses induced by a peptide-based cancer vaccine in a mouse model for HPV16-induced carcinoma (19). As a treatment option, administration of a monoclonal anti-NKG2A antibody to cancer patients would be more cost effective and logistically straightforward relative to cell therapy in which NK cells would need to be expanded and transduced under GMP conditions. However, NK numbers and function are frequently suppressed in patients with advanced cancer (21). Thus, the administration of blocking antibodies may not be effective for patients with very low cytotoxic lymphocyte numbers and/or defective cytotoxicity. Adoptive transfer of NKG2A ${ }^{\text {null }}$ NK cells, as suggested by Kamiya and colleagues, may be a promising therapy for cancers that are refractory to checkpoint inhibitory receptor blockade. It should be noted that, in order to efficiently transduce NK cells and generate large numbers of cells, the authors of the current study cocultured NK cells with the genetically modified K562-mbIL-15-41BBL cell line. What effects this potent stimulation might have on NK cell persistence and homing after adoptive transfer into humans are still largely unknown. Going forward, it is clear that NKG2A is an important inhibitory receptor on cytotoxic lymphocytes and that cancers can highly overexpress its HLA-E ligand. Future studies to compare the clinical benefit of utilizing $N K G 2 \mathrm{~A}^{\text {null }}$ cells generated by intracellular protein retention with strategies aimed at increasing CMV-induced NKG2 $\mathrm{C}^{+} \mathrm{NKG} 2 \mathrm{~A}^{\mathrm{lo} /-}$ adaptive NK cells or therapeutic antibody-mediated NKG2A blockade are needed.

\section{Acknowledgments}

We would like to acknowledge the following sources of $\mathrm{NIH}$ funding: K99/R00 HL123638 (to FC) and P01 CA111412, P01 CA65493, and R35 CA197292 (to JSM).

Address correspondence to: Jeffrey S. Miller, University of Minnesota, 420 Delaware Street SE, Minneapolis, Minnesota 55455, USA. Phone: 612.625.7409; Email: mille011@umn.edu.

1. Lazetic S, Chang C, Houchins JP, Lanier LL, Phillips JH. Human natural killer cell receptors involved in MHC class I recognition are disulfide-linked heterodimers of CD94 and NKG2 subunits. J Immunol. 1996;157(11):4741-4745.

2. Braud VM, et al. HLA-E binds to natural killer cell receptors CD94/NKG2A, B and C. Nature. 1998;391(6669):795-799.

3. Lee $\mathrm{N}$, et al. HLA-E is a major ligand for the natural killer inhibitory receptor CD94/NKG2A. Proc Natl Acad Sci U S A. 1998;95(9):5199-5204.

4. Paust S, Blish CA, Reeves RK. Redefining memory: building the case for adaptive NK cells. JVirol. 2017;91(20):e00169-17.

5. Gumá M, Angulo A, Vilches C, Gómez-Lozano N, Malats N, López-Botet M. Imprint of human cytomegalovirus infection on the NK cell receptor repertoire. Blood. 2004;104(12):3664-3671.

6. Le Dréan E, et al. Inhibition of antigen-induced $\mathrm{T}$ cell response and antibody-induced NK cell cytotoxicity by NKG2A: association of NKG2A with SHP-1 and SHP-2 protein-tyrosine phos- phatases. Eur J Immunol. 1998;28(1):264-276.

7. Stebbins CC, Watzl C, Billadeau DD, Leibson PJ, Burshtyn DN, Long EO. Vav1 dephosphorylation by the tyrosine phosphatase SHP-1 as a mechanism for inhibition of cellular cytotoxicity. $\mathrm{Mol}$ Cell Biol. 2003;23(17):6291-6299.

8. Liu D, Peterson ME, Long EO. The adaptor protein Crk control activation and inhibition of natural killer cells. Immunity. 2012;36(4):600-611.

9. Béziat V, Descours B, Parizot C, Debré P, Vieillard V. NK cell terminal differentiation: correlated stepwise decrease of NKG2A and acquisition of KIRs. PLoS One. 2010;5(8):e11966.

10. Braud V, Jones EY, McMichael A. The human major histocompatibility complex class Ib molecule HLA-E binds signal sequence-derived peptides with primary anchor residues at positions 2 and 9. Eur J Immunol. 1997;27(5):1164-1169.

11. Seliger B, et al. HLA-E expression and its clinical relevance in human renal cell carcinoma. Oncotarget. 2016;7(41):67360-67372.

12. Andersson E, et al. Non-classical HLA-class I expression in serious ovarian carcinoma: Correlation with the HLA-genotype, tumor infiltrating immune cells and prognosis. Oncoimmunology. 2015;5(1):e1052213.

13. Talebian Yazdi M, et al. The positive prognostic effect of stromal $\mathrm{CD}^{+}$tumor-infiltrating $\mathrm{T}$ cells is restrained by the expression of HLA-E in non-small cell lung carcinoma. Oncotarget. 2016;7(3):3477-3488.

14. Malmberg KJ, et al. IFN-gamma protects shortterm ovarian carcinoma cell lines from CTL lysis via a CD94/NKG2A-dependent mechanism. J Clin Invest. 2002;110(10):1515-1523.

15. Tomasec P, et al. Surface expression of HLA-E, an inhibitor of natural killer cells, enhanced by human cytomegalovirus gpUL40. Science. 2000;287(5455):1031.

16. Cichocki F, et al. CD56 ${ }^{\mathrm{dim}} \mathrm{CD} 57^{+} \mathrm{NKG} 2 \mathrm{C}^{+} \mathrm{NK}$ cell expansion is associated with reduced leukemia relapse after reduced intensity HCT. Leukemia. 2016;30(2):456-463.

17. Gooden M, et al. HLA-E expression by gynecological cancers restrains tumor-infiltrating CD8 ${ }^{+}$T lymphocytes. Proc Natl Acad Sci U S A. 2011;108(26):10656-10661.

18. Kamiya T, Seow SV, Wong D, Robinson M, Campana D. Blocking expression of inhibitory receptor NKG2A overcomes tumor resistance to NK cells. J Clin Invest. 2019;129(5):2094-2106.

19. van Montfoort N, et al. NKG2A blockade potentiates CD8 T cell immunity induced by cancer vaccines. Cell. 2018;175(7):1744-1755.e15.

20. André $\mathrm{P}$, et al. Anti-NKG2A mAb is a checkpoint inhibitor that promotes anti-tumor immunity by unleashing both T and NK cells. Cell. 2018;175(7):1731-1743.e13.

21. Introna M, Allavena P, Biondi A, Colombo N, Villa A, Mantovani A. Defective natural killer activity within human ovarian tumors: low numbers of morphologically defined effectors present in situ. J Natl Cancer Inst. 1983;70(1):21-26. 